\title{
A hybrid approach for the regularized long wave-Burgers equation
}

\author{
Asuman Zeytinoglu ${ }^{a}$, Murat Sari ${ }^{b^{*}}$, Bilender P. Allahverdiev ${ }^{a}$ \\ ${ }^{a}$ Department of Mathematics, Suleyman Demirel University, Turkey \\ ${ }^{b}$ Department of Mathematics, Yildiz Technical University, Turkey \\ asumanzeytinoglu@sdu.edu.tr, sarim@yildiz.edu.tr,bilenderpasaoglu@sdu.edu.tr
}

\section{ARTICLE INFO}

Article history:

Received: 13 March 2017

Accepted: 11 August 2017

Available Online: 10 October 2017

\section{Keywords:}

Regularized long wave-Burgers equation

High order finite difference scheme

Weighted essentially non-oscillatory

scheme

Hybrid approximation

MacCormack method

AMS Classification 2010:

65M06, 35Q35, 35G31

\begin{abstract}
In this paper, a new hybrid approach based on sixth-order finite difference and seventh-order weighted essentially non-oscillatory finite difference scheme is proposed to capture numerical simulation of the regularized long wave-Burgers equation which represents a balance relation among dissipation, dispersion and nonlinearity. The corresponding approach is implemented to the spatial derivatives and then MacCormack method is used for the resulting system. Some test problems discussed by different researchers are considered to apply the suggested method. The produced results are compared with some earlier studies, and to validate the accuracy and efficiency of the method, some error norms are computed. The obtained solutions are in good agreement with the literature. Furthermore, the accuracy of the method is higher than some previous works when some error norms are taken into consideration.
\end{abstract}

$((c))$ EY

\section{Introduction}

In describing many models in a great deal of fields of science, nonlinear partial differential equations (PDEs) play a significant role. Hence, reaching exact or well approximate solutions of nonlinear PDEs is still important. These kinds of partial differential equations may not have an exact solution by reason of their nonlinearity. So, it is of interest to introduce a new method or develop an existing technique to obtain accurate numerical results. One of the popular nonlinear partial differential equations studied for its numerical solutions is the regularized long waveBurgers (RLW-Burgers) equation also known as Benjamin-Bona-Mahony-Burgers (BBMB) equation. This equation describes the propagation of surface water waves in a channel [1]. The RLW-Burgers equation is considered as follows with physical boundary conditions $u \rightarrow 0$ as $x \rightarrow \pm \infty$ :

$u_{t}-u_{x x t}-\alpha u_{x x}+\beta u_{x}+g(u)_{x}=0,-\infty<x<\infty, t \geq 0$

$u(x, 0)=\phi(x) \rightarrow 0, \quad x \rightarrow \mp \infty$.

The subscripts $t$ and $x$ are time and space derivatives, and denote the horizontal coordinate along the channel and the elapsed time, respectively. $\phi(x)$ is a known function as initial condition, $\alpha$ is a positive constant, $\beta \in \square$ and $g(u)$ is a $C^{2}$ smooth nonlinear function. Eq. (1) represents a balance relation among dissipation, dispersion and nonlinearity [2]. Due to the fact that this equation is important for understanding the nonlinear wave phenomena, many researchers [29] have studied on it for many years.

Since the numerical methods are good means of understanding these types of equations, the effort of finding a more accurate numerical approach is still in progress. Investigating an effective and accurate numerical method encourages us to produce a new hybrid approach based on some high order finite difference (FD) schemes for analyzing the behavior of the RLW-Burgers equation. One of these FD schemes is a seventh-order weighted essentially non-oscillatory (WENO7) [10, 11, 16] method. It can be clear from the literature that the WENO method based on ENO schemes is one of the popular numerical methods for PDEs in conservative form $u_{t}+f(u)_{x}=0$. High order accuracy can be achieved in the smooth regions and discontinuities can be computed without spurious oscillations [12]. Some studies in recent years have introduced several versions of the WENO scheme derived for improving ENO properties [10-16]. However, some researchers have combined the WENO schemes with a high order method to overcome some drawbacks [17-19]. Inspired by these drawbacks in the corresponding studies, we prefer to 
combine the corresponding WENO scheme with the sixth-order finite difference (FD6) scheme [20, 21] because the FD6 gives convergent approximations as well as being effective, reliable and easy to implement. To validate the accuracy and efficiency of the proposed method, some error norms are presented and the obtained results are compared with the previous works in the literature.

The arrangement of this paper is as follows: The suggested scheme in both space and time are introduced in Section 2. Five test problems including different $\alpha, \beta$ parameters and $g(u), \phi(x)$ functions are solved to show the efficiency and accuracy of the proposed method, and the computed results are compared with others selected from the available literature in Section 3. Finally, the last section includes the summary of findings in the paper.

\section{Construction of the method}

One can rewrite problem (1) with the following form:

$$
u_{t}=v \text {, }
$$

$$
v_{x x}-v=f(u)_{x}-\alpha u_{x x}, f(u)_{x}=(\beta u+g(u))_{x} .
$$

As $\alpha, \beta$ and $g(u)$ change, Eq. (3) changes for each test problem. It can also be seen from the above system, there is no time derivative term in Eq. (3). The proposed approach is involved the FD6 and WENO7 finite difference formulations to the spatial derivatives, and the MacCormack discretization is taken into account for the time derivative. Details of the implementation of the present method are introduced in the following subsections.

\subsection{Space discretization with the hybrid scheme}

First of all, we divide the domain of problem $[a, b]$ into $N$ subintervals such as $a=x_{1}<x_{2}<\ldots<x_{N}<x_{N+1}=b$ with the spatial step size $h=\Delta x=x_{i+1}-x_{i}$ for $i=1,2 \ldots, N$. Also, $(n+1)$ th time level is defined by $t^{n+1}=t^{n}+\Delta t$ where $t^{n}$ is the initial time for $n=0$. Thus, the numerical solution of $u$ is represented by $u_{i}^{n}$ at grid point $\left(x_{i}, t^{n}\right)$. We use the FD6 scheme derived for the second order derivatives to discretize the terms $v_{x x}$ and $u_{x x}$ in Eq. (3). The FD6 scheme can briefly be introduced as follows:

$v^{\prime}$ and $v^{\prime \prime}$ in space, can be approximated by the following FD6 formulae used 7-point stencil

$$
v_{i}^{\prime}=\frac{1}{h} \sum_{j=-L}^{R} a_{j+L} v_{i+j}, \quad v_{i}^{\prime \prime}=\frac{1}{h^{2}} \sum_{j=-L}^{R} \tilde{a}_{j+L} v_{i+j}
$$

for $1 \leq i \leq N+1$.

In Equations (4), $(N+1)$ denotes the number of grid points, $\quad a_{k}$ and $\tilde{a}_{k}(k=0, \ldots, R+L)$ are unknown constants, $R$ and $L$ denote the number of grid points in the right and left hand side for the taken stencil, respectively. At internal points, $R$ and $L$ is equal while they are different for the boundary nodes. The coefficients $a_{k}$ and $\tilde{a}_{k}$ can be determined with Taylor series expansions about the related point and

\begin{tabular}{|c|c|c|c|c|c|c|c|c|}
\hline \multirow{2}{*}{$i$} & \multirow{2}{*}{ Coefficients* } & \multicolumn{7}{|c|}{$k$} \\
\hline & & $k=0$ & $k=1$ & $k=2$ & $k=3$ & $k=4$ & $k=5$ & $k=6$ \\
\hline \multirow{2}{*}{1} & $a_{k}$ & -147 & 360 & -450 & 400 & -225 & 72 & -10 \\
\hline & $\tilde{a}_{k}$ & 812 & -3132 & 5265 & -5080 & 2970 & -972 & 137 \\
\hline \multirow{2}{*}{2} & $a_{k}$ & -10 & -77 & 150 & -100 & 50 & -15 & 2 \\
\hline & $\tilde{a}_{k}$ & 137 & -147 & -255 & 470 & -285 & 93 & -13 \\
\hline \multirow{2}{*}{3} & $a_{k}$ & 2 & -24 & -35 & 80 & -30 & 8 & -1 \\
\hline & $\tilde{a}_{k}$ & -13 & 228 & -420 & 200 & 15 & -12 & 2 \\
\hline \multirow{2}{*}{$\begin{array}{c}\text { Internal } \\
\text { Nodes }\end{array}$} & $a_{k}$ & -1 & 9 & -45 & 0 & 45 & -9 & 1 \\
\hline & $\tilde{a}_{k}$ & 2 & -27 & 270 & -490 & 270 & -27 & 2 \\
\hline \multirow{2}{*}{$N-1$} & $a_{k}$ & 1 & -8 & 30 & -80 & 35 & 24 & -2 \\
\hline & $\tilde{a}_{k}$ & 2 & -12 & 15 & 200 & -420 & 228 & -13 \\
\hline \multirow{2}{*}{$N$} & $a_{k}$ & -2 & 15 & -50 & 100 & -150 & 77 & 10 \\
\hline & $\tilde{a}_{k}$ & -13 & 93 & -285 & 470 & -255 & -147 & 137 \\
\hline \multirow{2}{*}{$N+1$} & $a_{k}$ & 10 & -72 & 225 & -400 & 450 & -360 & 147 \\
\hline & $\tilde{a}_{k}$ & 137 & -972 & 2970 & -5080 & 5265 & -3132 & 812 \\
\hline
\end{tabular}
they are given in Table 1 .

Table 1. The coefficients $a_{k}$ and $\tilde{a}_{k}$

*Each given values of $a_{k}$ and $\tilde{a}_{k}$ in the table must be divided by 60 and 180 , respectively 
For the term $f(u)_{x}$ in Eq. (3), the WENO7 scheme is implemented together with the FD6 scheme. The WENO schemes are based on ENO schemes and it was first suggested by Liu et al [22]. They provide high order accurate solutions in smooth regions and have a good convergence since they use a convex combination of all candidate stencils against the ENO schemes. In the literature, many researchers have focused on the WENO schemes in order to improve them. Taking inspiration from those studies, the present work discusses a combination of the WENO7 finite difference scheme with the FD6 scheme in computing highly accurate results. The mentioned WENO scheme is applied to internal nodes and the FD6 formulae given in above are implemented for near the boundaries. We can then introduce the WENO7 procedure with its main points herein below $[10,11,16]$ :

The WENO schemes for discretization of the spatial derivatives in the following hyperbolic conservation law

$$
u_{t}+f(u)_{x}=0
$$

are successful in terms of the numerical approximation. A reconstruction procedure based on the local smoothness of numerical solution is used as the main point of the WENO finite difference scheme in order to produce high order accurate solutions. The term $f(u)_{x}$ is approximated by

$$
\left.f(u)_{x}\right|_{x=x_{j}} \approx \frac{1}{\Delta x}\left(\hat{f}_{j+\frac{1}{2}}-\hat{f}_{j-\frac{1}{2}}\right),
$$

where $\hat{f}_{j+\frac{1}{2}}$ represents the numerical flux. The WENO7 scheme uses 7 candidate stencils written as a set $S=\left\{x_{j-3}, \ldots, x_{j+3}\right\}$ for these numerical fluxes. It is

$$
\begin{aligned}
\beta_{0}= & f\left(u_{j-3}\right)\left[547 f\left(u_{j-3}\right)-3882 f\left(u_{j-2}\right)+4642 f\left(u_{j-1}\right)-1854 f\left(u_{j}\right)\right]+f\left(u_{j-2}\right)\left[7043 f\left(u_{j-2}\right)-17246 f\left(u_{j-1}\right)+7042 f\left(u_{j}\right)\right] \\
& +f\left(u_{j-1}\right)\left[11003 f\left(u_{j-1}\right)-9402 f\left(u_{j}\right)\right]+f\left(u_{j}\right)\left[2107 f\left(u_{j}\right)\right], \\
\beta_{1}= & f\left(u_{j-2}\right)\left[267 f\left(u_{j-2}\right)-1642 f\left(u_{j-1}\right)+1602 f\left(u_{j}\right)-494 f\left(u_{j+1}\right)\right]+f\left(u_{j-1}\right)\left[2843 f\left(u_{j-1}\right)-5966 f\left(u_{j}\right)+1922 f\left(u_{j+1}\right)\right] \\
& +f\left(u_{j}\right)\left[3443 f\left(u_{j}\right)-2522 f\left(u_{j+1}\right)\right]+f\left(u_{j+1}\right)\left[547 f\left(u_{j+1}\right)\right], \\
\beta_{2}= & f\left(u_{j-1}\right)\left[547 f\left(u_{j-1}\right)-2522 f\left(u_{j}\right)+1922 f\left(u_{j+1}\right)-494 f\left(u_{j+2}\right)\right]+f\left(u_{j}\right)\left[3443 f\left(u_{j}\right)-5966 f\left(u_{j+1}\right)+1602 f\left(u_{j+2}\right)\right] \\
& +f\left(u_{j+1}\right)\left[2843 f\left(u_{j+1}\right)-1642 f\left(u_{j+2}\right)\right]+f\left(u_{j+2}\right)\left[267 f\left(u_{j+2}\right)\right], \\
\beta_{3}= & f\left(u_{j}\right)\left[2107 f\left(u_{j}\right)-9402 f\left(u_{j+1}\right)+7042 f\left(u_{j+2}\right)-1854 f\left(u_{j+3}\right)\right]+f\left(u_{j+1}\right)\left[11003 f\left(u_{j+1}\right)-17246 f\left(u_{j+2}\right)+4642 f\left(u_{j+3}\right)\right] \\
& +f\left(u_{j+2}\right)\left[7043 f\left(u_{j+2}\right)-3882 f\left(u_{j+3}\right)\right]+f\left(u_{j+3}\right)\left[547 f\left(u_{j+3}\right)\right] .
\end{aligned}
$$

Table 2. The coefficients $b_{m i}$ for the WENO7 scheme

\begin{tabular}{c|cccc}
\hline$b_{m i}$ & $i=0$ & $i=1$ & $i=2$ & $i=3$ \\
\hline$m=0$ & $25 / 12$ & $-23 / 12$ & $13 / 12$ & $-1 / 4$ \\
$m=1$ & $1 / 4$ & $13 / 12$ & $-5 / 12$ & $1 / 12$ \\
$m=2$ & $-1 / 12$ & $7 / 12$ & $7 / 12$ & $-1 / 12$ \\
$m=3$ & $1 / 12$ & $-5 / 12$ & $13 / 12$ & $1 / 4$ \\
\hline
\end{tabular}


For more details of the WENO finite difference scheme, interested readers are referred to as the literature such as [10-13, 16].

\subsection{Time discretization with MacCormack method}

After the implementation of the aforementioned main schemes to Eq. (3), the values of variable $v$ are found and then the MacCormack method is used to find new values of $u$ at the next time level from Eq. (2). This method is widely used for solving nonlinear PDEs representing fluid flows and provides accurate results [24]. Let us consider the following general form of governing equation

$$
\frac{d u_{i}}{d t}=P u_{i}
$$

In this form, $P$ represents a spatial differential operator, and each values on the right hand side of the above equation are already known through the method described in the previous subsection. In order to solve this semi-discrete equation, the MacCormack approach is then implemented via the following process:

$$
\text { Pre. Step: } u_{i}^{\overline{n+1}}=u_{i}^{n}+\Delta t P u_{i}^{n} \text {, }
$$

Cor. Step: $u_{i}^{n+1}=u_{i}^{n+1 / 2}+\frac{\Delta t}{2} P u_{i}^{\overline{n+1}}, u_{i}^{n+1 / 2}=\frac{u_{i}^{n}+u_{i}^{\overline{n+1}}}{2}$.

\section{Numerical Illustrations}

In this section, we implement the previous procedure to five test problems for producing numerical solutions of the RLW-Burgers equation. The accuracy of the numerical solutions is observed by using absolute error and the following error norms

$$
\begin{gathered}
L_{2}=\sqrt{h \sum_{j=1}^{N}\left|u_{j}^{\text {analytical }}-u_{j}^{\text {numerical }}\right|^{2}}, \\
L_{\infty}=\max \left|u_{j}^{\text {analytical }}-u_{j}^{\text {numerical }}\right| .
\end{gathered}
$$

which measure the mean and maximum differences between the numerical and analytical solutions. To show the behaviors of corresponding problems, some figures are also plotted.

Example 1. As the first test problem, Eq. (1) with $\alpha=1, \beta=1$ and $g(u)=u^{2} / 2$ is considered by the following initial condition

$$
u(x, 0)=\operatorname{sech}^{2}(x / 4) .
$$

Table 3 gives the obtained results using $h=0.25$ and $\Delta t=0.01$ in the interval $-12 \leq x \leq 12$. It can be clearly seen that the produced results are compatible with the results of Zarebnia and Parvaz [8]. Also, the solutions at various times are qualitatively presented in Figure 1. As is the case in the study of Zarebnia and Parvaz [8] and as naturally expected, the amplitude of wave slightly decreases as the time goes on (see Figure 1).

Table 3. Numerical results with the parameters $h=0.25$ and $\Delta t=0.01$ for Example 1

\begin{tabular}{ccccccc}
\hline & \multicolumn{6}{c}{ Present method } \\
\cline { 2 - 7 }$x$ & \multicolumn{7}{c}{$t$} \\
\cline { 2 - 7 }-10 & 0.024700 & 0.022144 & 0.020606 & 0.018518 & 0.015529 & 0.013038 \\
-5 & 0.256292 & 0.224794 & 0.206475 & 0.182379 & 0.149529 & 0.123687 \\
0 & 0.978142 & 0.933383 & 0.897619 & 0.838349 & 0.733532 & 0.631524 \\
5 & 0.319370 & 0.380986 & 0.423415 & 0.487534 & 0.589834 & 0.676862 \\
10 & 0.032031 & 0.041918 & 0.049799 & 0.063792 & 0.093622 & 0.132550 \\
\hline & & \multicolumn{5}{c}{1.5} \\
\hline-10 & 0.022951 & 0.019822 & 0.017950 & 0.015435 & 0.011934 & 0.009165 \\
-5 & 0.256278 & 0.224742 & 0.206391 & 0.182231 & 0.149239 & 0.123215 \\
0 & 0.978102 & 0.933352 & 0.897596 & 0.83834 & 0.733537 & 0.631526 \\
5 & 0.319376 & 0.380993 & 0.42342 & 0.487532 & 0.589817 & 0.676809 \\
10 & 0.030420 & 0.039796 & 0.047263 & 0.060502 & 0.088659 & 0.12528 \\
\hline
\end{tabular}

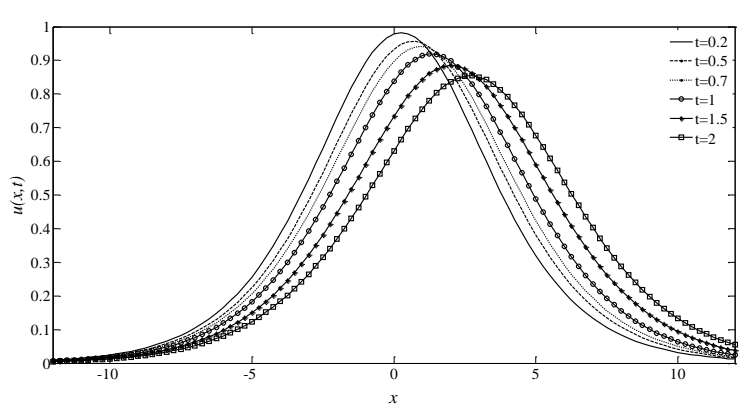

Figure 1. The behavior of the wave in Example 1.
Example 2. Consider Eq. (1) with the initial condition

$$
u(x, 0)=-\frac{9}{5}-\frac{6}{5} \tanh \left(\frac{x}{2}\right)+\frac{3}{5} \tanh ^{2}\left(\frac{x}{2}\right)
$$

using $\alpha=1, \beta=1$ and $g(u)=u^{2} / 2$. The analytical solution is given by

$$
u(x, t)=-\frac{9}{5}-\frac{6}{5} \tanh \left(\frac{5 x+t}{10}\right)+\frac{3}{5} \tanh ^{2}\left(\frac{5 x+t}{10}\right) .
$$

For this problem the parameters are chosen as $h=0.2$ and $\Delta t=0.01$ in the interval $-32 \leq x \leq 32$. In Table 4 , 
the produced $L_{2}, L_{\infty}$ errors are given. The obtained error values are quite good even for the larger time $t=10$. Furthermore, absolute errors at various points in the corresponding domain are presented and compared with the study of Alquran and Al-Khaled [4] for some time values in Table 5. It can be said that our results are at least three decimal digits better than the results of Alquran and Al-Khaled [4]. The qualitative behavior of solutions at $t=10$ and at various times are exhibited in Figure 2(a)-2(b), respectively.

Table 4. $L_{2}$ and $L_{\infty}$ error norms for Example 2

\begin{tabular}{lcc}
\hline$t$ & $L_{2}$ & $L_{\infty}$ \\
\hline 0.2 & $1.826363 \mathrm{E}-07$ & $1.678531 \mathrm{E}-07$ \\
0.4 & $3.371454 \mathrm{E}-07$ & $3.068812 \mathrm{E}-07$ \\
1 & $6.734666 \mathrm{E}-07$ & $6.326276 \mathrm{E}-07$ \\
3 & $1.096361 \mathrm{E}-06$ & $9.812815 \mathrm{E}-07$ \\
10 & $1.754436 \mathrm{E}-05$ & $1.546722 \mathrm{E}-05$ \\
\hline
\end{tabular}

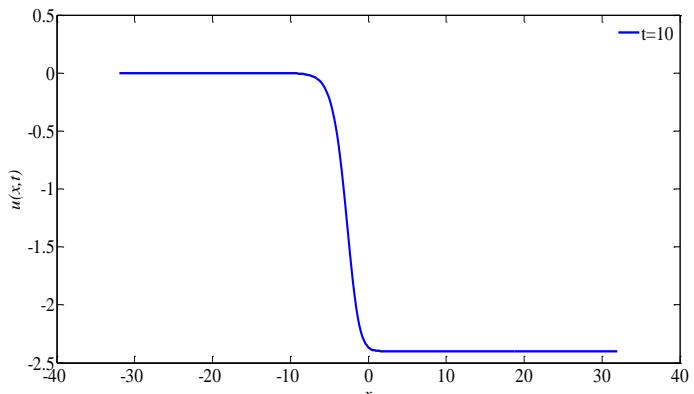

(a)

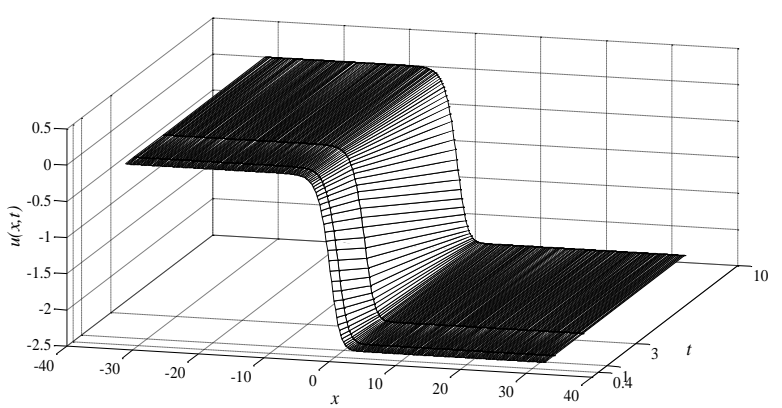

(b)

Figure 2. Numerical solutions for Example 2 using $h=0.2$ and $\Delta t=0.01$ for (a) $t=10$ and (b) various times.

Table 5. Absolute errors at various times for Example 2

\begin{tabular}{c|ccccccc}
\hline \multirow{2}{*}{$x \backslash t$} & $\begin{array}{c}\text { Present } \\
\text { Method }\end{array}$ & {$[4]$} & $\begin{array}{c}\text { Present } \\
\text { Method }\end{array}$ & {$[4]$} & $\begin{array}{c}\text { Present } \\
\text { Method }\end{array}$ & $\begin{array}{c}\text { Present } \\
\text { Method }\end{array}$ & $\begin{array}{c}\text { Present } \\
\text { Method }\end{array}$ \\
\cline { 2 - 8 }$y$ & 0.2 & 0.2 & 0.4 & 0.4 & 1 & 3 & 10 \\
\hline 0.2 & $1.28 \mathrm{E}-07$ & $6.76 \mathrm{E}-04$ & $2.22 \mathrm{E}-07$ & $5.02 \mathrm{E}-04$ & $3.39 \mathrm{E}-07$ & $7.71 \mathrm{E}-08$ & $1.92 \mathrm{E}-07$ \\
0.4 & $6.42 \mathrm{E}-08$ & $5.05 \mathrm{E}-04$ & $1.00 \mathrm{E}-07$ & $4.52 \mathrm{E}-04$ & $9.16 \mathrm{E}-08$ & $2.84 \mathrm{E}-07$ & $2.11 \mathrm{E}-07$ \\
0.6 & $1.63 \mathrm{E}-09$ & $4.82 \mathrm{E}-04$ & $1.16 \mathrm{E}-08$ & $6.02 \mathrm{E}-04$ & $1.02 \mathrm{E}-07$ & $3.27 \mathrm{E}-07$ & $1.97 \mathrm{E}-07$ \\
0.8 & $4.01 \mathrm{E}-08$ & $4.14 \mathrm{E}-04$ & $8.19 \mathrm{E}-08$ & $6.02 \mathrm{E}-04$ & $1.97 \mathrm{E}-07$ & $2.47 \mathrm{E}-07$ & $1.65 \mathrm{E}-07$ \\
1 & $5.50 \mathrm{E}-08$ & $3.21 \mathrm{E}-04$ & $1.03 \mathrm{E}-07$ & $2.02 \mathrm{E}-04$ & $1.98 \mathrm{E}-07$ & $1.12 \mathrm{E}-07$ & $1.26 \mathrm{E}-07$ \\
1.2 & $4.85 \mathrm{E}-08$ & $6.05 \mathrm{E}-05$ & $8.56 \mathrm{E}-08$ & $7.51 \mathrm{E}-05$ & $1.39 \mathrm{E}-07$ & $1.90 \mathrm{E}-08$ & $8.94 \mathrm{E}-08$ \\
1.4 & $3.07 \mathrm{E}-08$ & $5.85 \mathrm{E}-05$ & $5.05 \mathrm{E}-08$ & $5.98 \mathrm{E}-05$ & $6.02 \mathrm{E}-08$ & $1.14 \mathrm{E}-07$ & $5.81 \mathrm{E}-08$ \\
1.8 & $4.73 \mathrm{E}-09$ & $1.36 \mathrm{E}-05$ & $1.39 \mathrm{E}-08$ & $2.37 \mathrm{E}-05$ & $5.65 \mathrm{E}-08$ & $1.76 \mathrm{E}-07$ & $1.66 \mathrm{E}-08$ \\
2.4 & $1.96 \mathrm{E}-08$ & $1.25 \mathrm{E}-05$ & $3.75 \mathrm{E}-08$ & $1.01 \mathrm{E}-05$ & $7.90 \mathrm{E}-08$ & $1.08 \mathrm{E}-07$ & $5.72 \mathrm{E}-09$ \\
3 & $1.20 \mathrm{E}-08$ & $6.23 \mathrm{E}-06$ & $2.19 \mathrm{E}-08$ & $7.78 \mathrm{E}-06$ & $4.08 \mathrm{E}-08$ & $3.76 \mathrm{E}-08$ & $7.24 \mathrm{E}-09$ \\
5 & $3.92 \mathrm{E}-10$ & $4.82 \mathrm{E}-06$ & $6.01 \mathrm{E}-10$ & $6.42 \mathrm{E}-06$ & $4.69 \mathrm{E}-10$ & $2.01 \mathrm{E}-09$ & $6.30 \mathrm{E}-10$ \\
\hline
\end{tabular}

Example 3. We now consider Eq. (1) with the parameters $\alpha=1, \beta=1$ and $g(u)=u^{2} / 2$ with the initial condition

$$
u(x, 0)=\exp \left(-x^{2}\right) .
$$

In this example, the domain is taken to be $-30 \leq x \leq 30$ and the behavior of the problem is examined up to time $t=10$. We use $h=0.2$ and $\Delta t=0.1$ in the proposed scheme and the recorded values are presented in Table 6. Furthermore, the profile of the wave is plotted in Figure 3 from $t=0$ to $t=10$. It can be seen from the figure that the amplitude of wave and the position of that amplitude changes in time. The amplitude of wave is equal to 1 located at $x=0$ for initial time, while that value decreases as the times goes on and it becomes about 0.2 located close by $x=10$.

Table 6. Numerical results for Example 3 with $h=0.2$ and

\begin{tabular}{c|crrr}
\multicolumn{5}{c}{$\Delta t=0.1$} \\
\hline$x \backslash t$ & 1 & 2 & 5 & 10 \\
\hline-30 & $-5.100 \mathrm{E}-13$ & $-3.027 \mathrm{E}-12$ & $-6.602 \mathrm{E}-11$ & $-1.174 \mathrm{E}-09$ \\
-15 & $-4.952 \mathrm{E}-08$ & $-4.754 \mathrm{E}-08$ & $-1.567 \mathrm{E}-08$ & $-1.462 \mathrm{E}-09$ \\
0 & $5.726 \mathrm{E}-01$ & $2.871 \mathrm{E}-01$ & $2.032 \mathrm{E}-02$ & $-2.175 \mathrm{E}-03$ \\
15 & $2.199 \mathrm{E}-05$ & $2.396 \mathrm{E}-04$ & $8.813 \mathrm{E}-03$ & $9.039 \mathrm{E}-02$ \\
30 & $1.653 \mathrm{E}-10$ & $5.346 \mathrm{E}-09$ & $2.186 \mathrm{E}-06$ & $4.348 \mathrm{E}-04$ \\
\hline
\end{tabular}




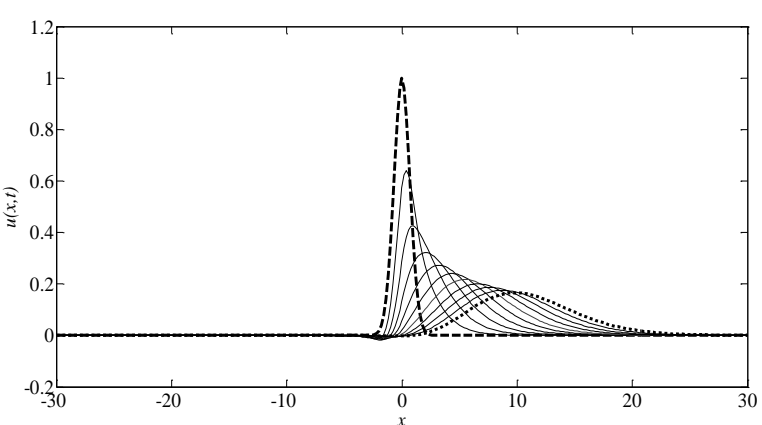

Figure 3. The behavior of the wave in Example 3 from $t=0$ to $t=10$ using $h=0.2$ and $\Delta t=0.1$.

Example 4. As the fourth test problem, Eq. (1) under the consideration of parameters $\alpha=1, \quad \beta=1$ and $g(u)=6 u^{2}$ is studied using the following initial condition

$$
u(x, 0)=-\frac{23}{120}-\frac{1}{5} \tanh x+\frac{1}{10} \tanh ^{2} x
$$

extracted from the exact solution

$$
u(x, t)=-\frac{23}{120}-\frac{1}{5} \tanh \left(x+\frac{t}{10}\right)+\frac{1}{10} \tanh ^{2}\left(x+\frac{t}{10}\right) .
$$

For comparison with an early work by Zhao et al. [2], $L_{\infty}$ errors are calculated for various times over the domain $-50 \leq x \leq 50$. The parameter $h$ is taken to be 0.2 with both $\Delta t=0.01$ and $\Delta t=0.1$, and the results are presented up to time $t=10$ in Table 7 . It is seen that the obtained $L_{\infty}$ errors are less than the compared results. Furthermore, the presented errors still decrease when $\Delta t=0.01$. The behavior of the problem for three different times are given in Figure 4.

\begin{tabular}{|c|c|c|c|}
\hline \multirow[b]{3}{*}{$t$} & \multicolumn{3}{|c|}{$L_{\infty}$} \\
\hline & \multicolumn{2}{|c|}{ Present Method } & [2] \\
\hline & $\begin{array}{c}h=0.2, \\
\Delta t=0.01\end{array}$ & $h=0.2$, & $h=0.2$ \\
\hline 0.2 & $6.048 \mathrm{E}-06$ & $6.553 \mathrm{E}-06$ & $7.650 \mathrm{E}-05$ \\
\hline 0.3 & $9.310 \mathrm{E}-06$ & $1.003 \mathrm{E}-05$ & $6.954 \mathrm{E}-05$ \\
\hline 0.4 & $1.274 \mathrm{E}-05$ & $1.365 \mathrm{E}-05$ & $1.490 \mathrm{E}-04$ \\
\hline 0.5 & $1.632 \mathrm{E}-05$ & $1.739 \mathrm{E}-05$ & $1.334 \mathrm{E}-04$ \\
\hline 0.6 & $2.001 \mathrm{E}-05$ & $2.123 \mathrm{E}-05$ & $2.160 \mathrm{E}-04$ \\
\hline 0.7 & 2.377E-05 & $2.510 \mathrm{E}-05$ & $1.918 \mathrm{E}-04$ \\
\hline 0.8 & 2.752E-05 & $2.895 \mathrm{E}-05$ & $2.774 \mathrm{E}-04$ \\
\hline 0.9 & $3.119 \mathrm{E}-05$ & $3.270 \mathrm{E}-05$ & $2.474 \mathrm{E}-04$ \\
\hline 1 & $3.472 \mathrm{E}-05$ & $3.629 \mathrm{E}-05$ & $3.385 \mathrm{E}-04$ \\
\hline 2 & $5.823 \mathrm{E}-05$ & $6.143 \mathrm{E}-05$ & - \\
\hline 3 & $9.216 \mathrm{E}-05$ & $9.493 \mathrm{E}-05$ & - \\
\hline 5 & $1.481 \mathrm{E}-04$ & $1.511 \mathrm{E}-04$ & - \\
\hline 10 & $2.871 \mathrm{E}-04$ & $2.889 \mathrm{E}-04$ & - \\
\hline
\end{tabular}

Table 7. $L_{\infty}$ errors at various times for Example 4 using

Example 5. For the last problem, the nonlinear function $g(u)$ is chosen as $u^{3} / 3, u^{5} / 5$ and $u^{9} / 9$, respectively, for Eq. (1) with $\alpha=1 / 2, \beta=1$.

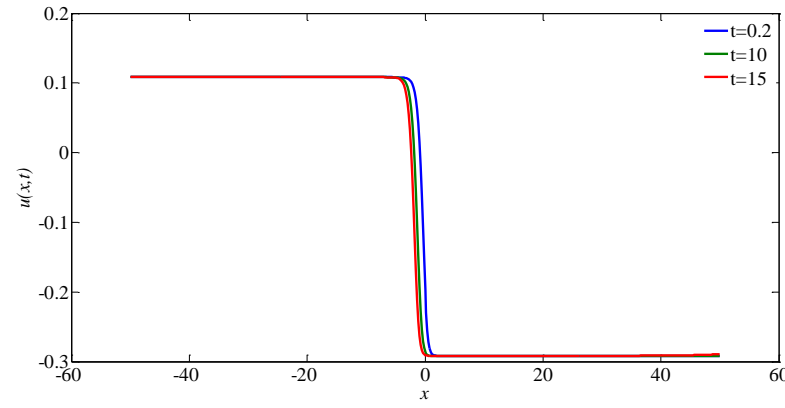

Figure 4. Numerical solutions for Example 4 at different times.

The initial condition is taken to be

$$
u(x, 0)=1 /\left(1+x^{4}\right) .
$$

In this example, the solutions with considering various nonlinear function $g(u)$ are investigated up to time $t=10$. In Figure 5, the solutions are plotted at different times using $h=0.2$ and $\Delta t=0.1$. To show the effect of parameter $h$, the solutions at $t=10$ are also displayed in Figure 6 using $\Delta t=0.1$ and various $h$ values. It is observed from the corresponding figures that due to the value of $\alpha$ parameter, a slight oscillation occurs at the beginning of the wave, and the amplitude of both wave and oscillation decreases as the time goes on. To see the effect of $\alpha$ parameter on the behavior of the wave, Figure 7 is presented for various values of $\alpha$. In the calculations, the parameters are taken to be $h=0.2, \Delta t=0.1$, $g(u)=u^{3} / 3$. Figure 7 shows that as the value of $\alpha$ decreases, the amplitude of the wave slightly increases. However, any oscillation does not appear in the wave motion if $\alpha$ value is taken larger. It can be also seen that as the $\alpha$ value decreases, some oscillations occur. The results in the above examples revealed that the proposed method has been seen to be usually more convergent and easier than its rival methods from the literature.

\section{Conclusion}

A hybrid approach based on two different types of finite difference scheme has been introduced and applied for the solutions of some physical problems constructed with the RLW-Burgers equation. To reveal the accuracy of the proposed scheme, five test problems are considered for various values of parameters taken part in the RLW-Burgers equation, and some error norms, such as absolute, $L_{2}$ and $L_{\infty}$, are presented. The computed results revealed that the suggested method highly accurate, computationally powerful and user-friendly. The present approach is also believed to be easier in producing computer codes for applications. Therefore, it is seen to be a strongly advisable alternative to discover both qualitative and quantitative behaviors of similar processes for further studies. 


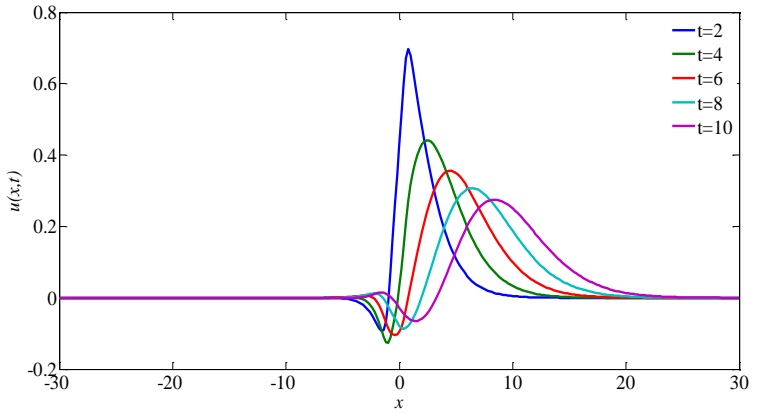

(a) $g(u)=u^{3} / 3$

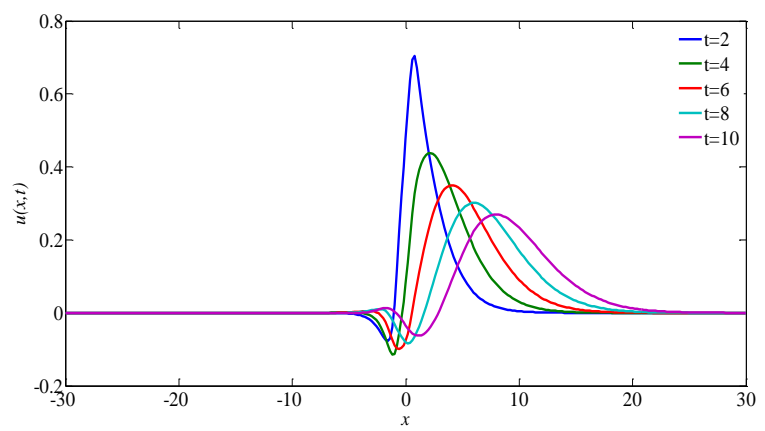

(b) $g(u)=u^{5} / 5$

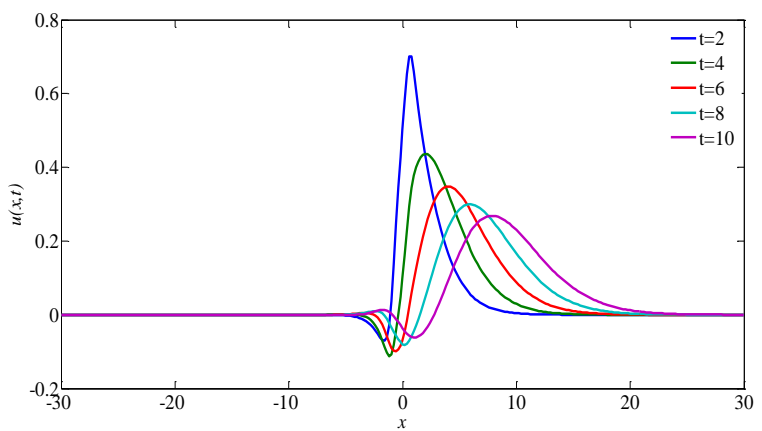

(c) $g(u)=u^{9} / 9$

Figure 5. The behaviors of the wave in Example 5 using $h=0.2, \Delta t=0.1$ for various $g(u)$

\section{Acknowledgments}

This research was supported by Suleyman Demirel University through Scientific Research Project Program (3539-D1-13).

\section{References}

[1] Bona, J.L., Pritchard, W.G. \& Scott, L.R. (1981). An evaluation of a model equation for water waves. Philosophical Transactions of the Royal Society of London. Series A, Mathematical and Physical Sciences, 302(1471), 457-510.

[2] Zhao, X., Li, D. \& Shi, D. (2008). A finite difference scheme for RLW-Burgers equation. Journal of Applied Mathematics \& Informatics, 26(3,4), 573581.

[3] Al-Khaled, K., Momani, S. \& Alawneh, A. (2005).

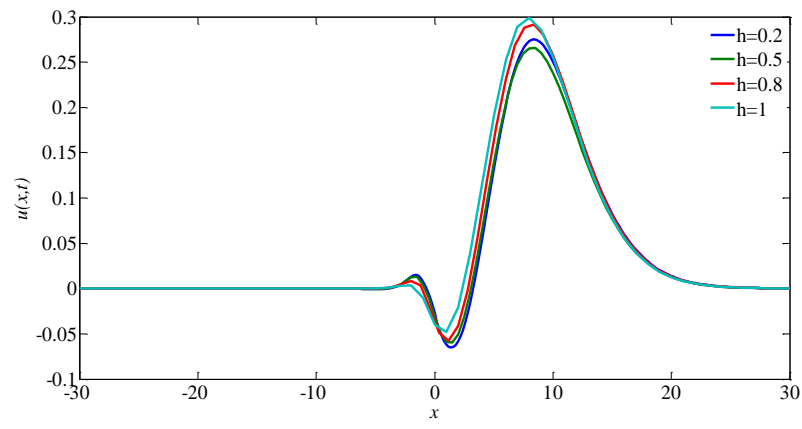

(a) $g(u)=u^{3} / 3$

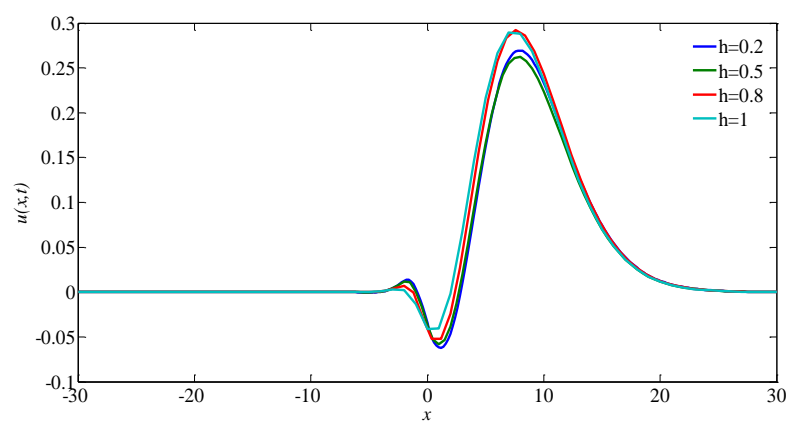

(b) $g(u)=u^{5} / 5$

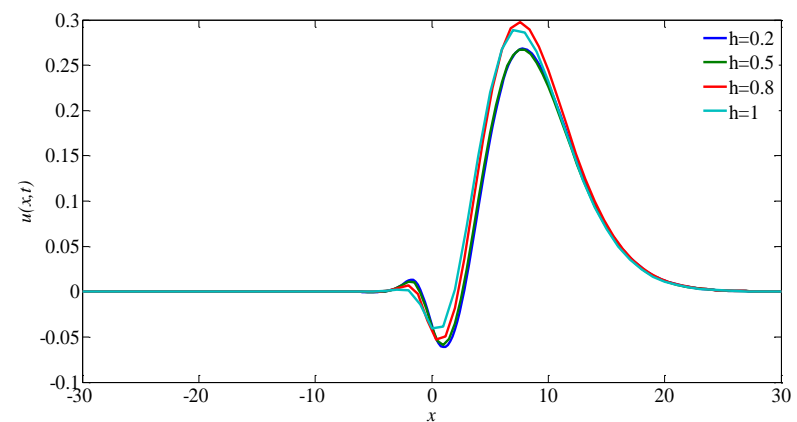

(c) $g(u)=u^{9} / 9$

Figure 6. Numerical solutions for Example 5 at $t=10$ using $\Delta t=0.1$ and various values of $h$ with various $g(u)$

Approximate wave solutions for generalized Benjamin-Bona-Mahony-Burgers equations. Applied Mathematics and Computation, 171(1), 281-292.

[4] Alquran, M. \& Al-Khaled, K. (2011). Sinc and solitary wave solutions to the generalized BenjaminBona-Mahony-Burgers equations. Physica Scripta, 83(6), 6 pages.

[5] Arora, G., Mittal, R.C. \& Singh, B.K. (2014). Numerical solution of BBM-Burgers equation with quartic B-spline collocation method. Journal of Engineering Science and Technology, 9, 104-116.

[6] Che, H., Pan, X., Zhang, L. \& Wang, Y. (2012). Numerical analysis of a linear-implicit average scheme for generalized Benjamin-Bona-MahonyBurgers equation. Journal of Applied Mathematics, 2012, 14 pages. 


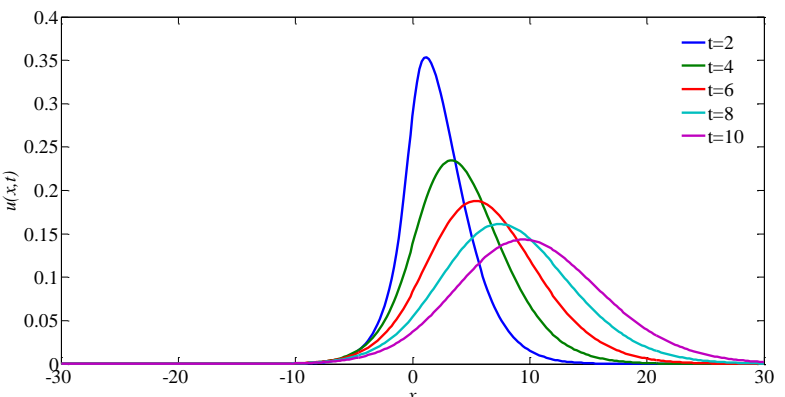

(a) $\alpha=2$

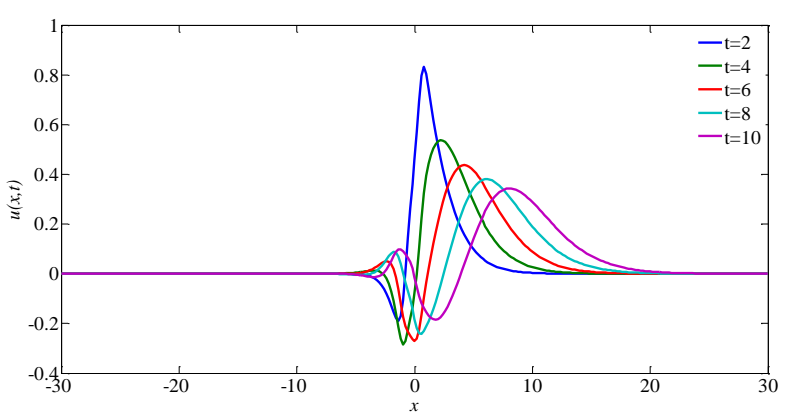

(c) $\alpha=0.25$

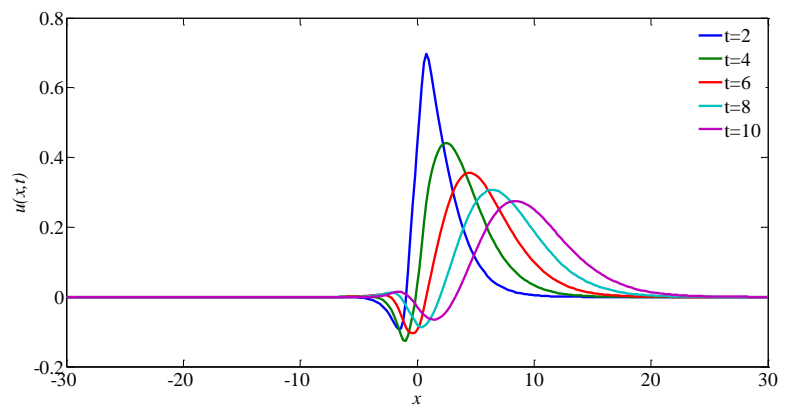

(b) $\alpha=0.5$

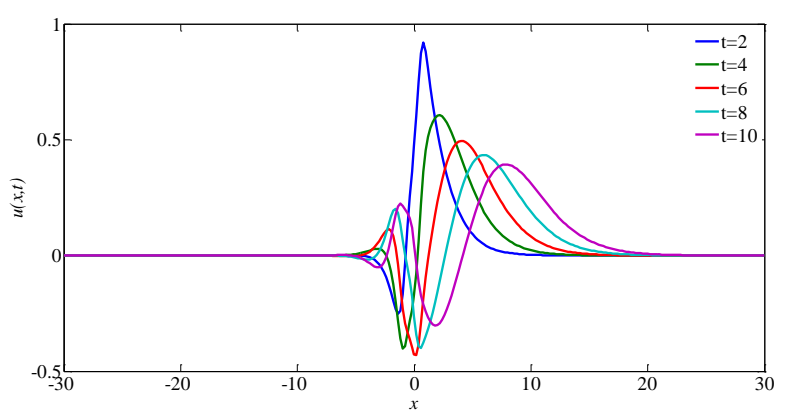

(d) $\alpha=0.125$

Figure 7. The behaviors of the wave in Example 5 using $h=0.2, \Delta t=0.1$ and $g(u)=u^{3} / 3$ for various values of $\alpha$.

[7] Omrani, K. \& Ayadi, M. (2008). Finite difference discretization of the Benjamin-Bona-MahonyBurgers equation. Numerical Methods for Partial Differential Equations, 24(1), 239-248.

[8] Zarebnia, M. \& Parvaz, R. (2013). Cubic B-spline collocation method for numerical solution of the Benjamin-Bona-Mahony-Burgers equation. WASET International Journal of Mathematical, Computational, Physical, Electrical and Computer Engineering, 7(3), 540-543.

[9] Zarebnia, M. \& Parvaz, R. (2016). On the numerical treatment and analysis of BenjaminBona-Mahony-Burgers equation. Applied Mathematics and Computation, 284, 79-88.

[10] Shen, Y. \& Zha, G. (2008). A Robust seventh-order WENO scheme and its applications, 46th AIAA Aerospace Sciences Meeting and Exhibit, 2008 Jan 7-10, Reno, Nevada, AIAA 2008-075.

[11] Shen, Y. \& Zha, G. (2010). Improved seventh order WENO scheme. 48th AIAA Aerospace Sciences Meeting Including the New Horizons Forum and Aerospace Exposition, 2010 Jan 4-7, Orlando, Florida, AIAA 2010-1451.

[12] Zahran, Y.H. \& Babatin, M.M. (2013). Improved ninth order WENO scheme for hyperbolic conservation laws. Applied Mathematics and Computation, 219(15), 8198-8212.

[13] Jiang, G.S. \& Shu, C.W. (1996). Efficient implementation of weighted ENO schemes. Journal of Computational Physics, 126(1), 202-228.
[14] Wang, Z.J. \& Chen, R.F. (2001). Optimized weighted essentially non-oscillatory schemes for linear waves with discontinuity. Journal of Computational Physics, 174(1), 381-404.

[15] Ponziani, D., Prizzoli, S. \& Grasso, F. (2003). Develpoment of optimized weighted-ENO schemes for multiscale compressible flows. International Journal for Numerical Methods in Fluids, 42(9), 953-977.

[16] Balsara, D.S. \& Shu, C.W. (2000). Monotonicity preserving weighted essentially non-oscillatory schemes with increasingly high order of accuracy. Journal of Computational Physics, 160(2), 405452 .

[17] Pirozzoli, S. (2002). Conservative hybrid compactWENO schemes for shock-turbulence interaction. Journal of Computational Physics, 178(1), 81-117.

[18] Kim, D. \& Kwon, J.H. (2005). A high-order accurate hybrid scheme using a central flux scheme and a WENO scheme for compressible flowfield analysis. Journal of Computational Physics, 210(2), 554-583.

[19] Shen, Y.Q. \& Yang, G.W. (2007). Hybrid finite compact WENO schemes for shock calculation. International Journal for Numerical Methods in Fluids, 53(4), 531-560.

[20] Sari, M., Gürarslan, G. \& Zeytinoglu, A. (2010). High-order finite difference schemes for solving the advection diffusion equation. Mathematical and Computational Applications, 15(3), 449-460. 
[21] Zeytinoglu, A. (2010). Some approximate solutions of Burgers equations. MSc Thesis. Suleyman Demirel University.

[22] Liu, X.D., Osher, S. \& Chan, T. (1994). Weighted essentially non-oscillatory schemes. Journal of Computational Physics, 126, 200-212.

[23] Xie, P., (2007). Uniform weighted compact/noncompact schemes for shock/boundary layer interaction. $\mathrm{PhD}$ Thesis. The University of Texas.

[24] Pletcher, R.H., Tannehill, J.C. \& Anderson, D.A. (2013). Computational fluid mechanics and fluid transfer. Taylor\&Francis.

Asuman Zeytinoglu is a Research Assistant in the Department of Mathematics at Suleyman Demirel University, Isparta, Turkey. She received her BSc degree in Mathematics from Pamukkale University, Denizli, Turkey in 2007, and MSc degree in Mathematics from Suleyman Demirel University, Isparta, Turkey in 2010. She is currently continuing the PhD studies in Department of Mathematics at Suleyman Demirel University, Isparta, Turkey. Her research interests are numerical solutions of partial differential equations, computational methods and wave propagation.

Murat Sari is an Associate Professor in the Department of Mathematics at Yildiz Technical University, Istanbul, Turkey. He received his BSc degree from Ondokuzmayis University, Turkey in 1991. He completed his graduate studies (MSc/PhD degrees) in Mathematics from University of Glamorgan, UK in 2000. His research interests are numerical methods, wave propagation, computational methods, mathematical modelling, biophysical modelling, medical modelling and economical modelling.

Bilender P. Allahverdiev is a Professor in the Department of Mathematics at Suleyman Demirel University, Isparta, Turkey. He received his BSc degree in Mathematics from Baku State University, Baku, Azerbaijan in 1977. He received his PhD degree from Azerbaijan National Academy of Sciences, Azerbaijan in 1982. His research interests are operator theory, spectral theory of differential operators, Jacobian matrix and difference equations, Schrödinger equation and Sturm-Liouville boundary value problems.

An International Journal of Optimization and Control: Theories \& Applications (http://ijocta.balikesir.edu.tr)

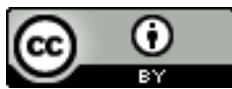

This work is licensed under a Creative Commons Attribution 4.0 International License. The authors retain ownership of the copyright for their article, but they allow anyone to download, reuse, reprint, modify, distribute, and/or copy articles in IJOCTA, so long as the original authors and source are credited. To see the complete license contents, please visit http://creativecommons.org/licenses/by/4.0/. 